\title{
M. pneumoniae and C. pneumoniae are no relevant pathogens in critically ill patients with hospital-acquired respiratory tract infections
}

\author{
Stefan Hagel ${ }^{1}(1) \cdot$ Svenja Schmitt $^{1} \cdot$ Miriam Kesselmeier $^{2} \cdot$ Michael Baier $^{3} \cdot$ Tobias Welte $^{4} \cdot$ Santiago $_{\text {Ewig }^{5}}$. \\ Mathias W. Pletz ${ }^{1}$
}

Received: 16 November 2018 / Accepted: 17 January 2019 / Published online: 28 January 2019

(c) Springer-Verlag GmbH Germany, part of Springer Nature 2019

\begin{abstract}
Purpose To assess the incidence of Mycoplasma pneumoniae and Chlamydia pneumoniae in the pathogenesis of hospitalacquired respiratory tract infections (RTIs) in critically ill patients.

Methods This is a retrospective cohort study of all ICU-patients $\geq 18$ years with RTI who underwent conventional culture techniques and PCR testing for both M. pneumoniae and C. pneumoniae from respiratory tract specimens (bronchoalveolar lavage or tracheobronchial aspirates) between January 2013 to May 2017 at the Jena University Hospital.

Results In total, 314 patients were included in the analysis. Of these, $210(66.9 \%)$ patients were diagnosed with HAP, 65 (20.7\%) with VAP and 39 (12.4\%) with VAT. Overall, 73 (30.7\%) patients were on mechanical ventilation on the day of microbiological examination. PCR-testing for M. pneumoniae was positive in two patients $(0.6 \%)$ and for $C$. pneumoniae in zero patients.

Conclusions Our study shows that the incidence of M. pneumoniae and C. pneumoniae in the pathogenesis of hospitalacquired RTIs in critically ill patients is negligible. The results support the recommendations of the guidelines not to perform empiric therapy covering these pathogens.
\end{abstract}

Keywords Hospital-acquired pneumonia (HAP) $\cdot$ Ventilator-associated pneumonia (VAP) $\cdot$ Ventilator-associated tracheobronchitis (VAT) $\cdot$ Mycoplasma pneumoniae $\cdot$ Chlamydia pneumoniae

\section{Introduction}

Stefan Hagel and Svenja Schmitt contributed equally.

Stefan Hagel

stefan.hagel@med.uni-jena.de

1 Institute for Infectious Diseases and Infection Control, Jena University Hospital, Am Klinikum 1, 07747 Jena, Germany

2 Research Group Clinical Epidemiology, Center for Sepsis Control and Care (CSCC), University Hospital, Jena, Germany

3 Institute of Medical Microbiology, Jena University Hospital, Jena, Germany

4 Department of Respiratory Medicine, Hannover Medical School, Hannover, Germany

5 Department of Respiratory Medicine and Infectious Diseases, Thorax Centre in the Ruhr Area, Evangelic Hospital in Herne and Augusta Hospital in Bochum, Bochum, Germany
Guidelines for the management of hospital-acquired pneumonia (HAP) and ventilator-associated pneumonia (VAP) in adults do not recommend specific microbiological testing or empiric antimicrobial therapy for Mycoplasma pneumoniae and Chlamydia pneumoniae [1-3]. However, underlying data supporting this recommendation are sparse. M. pneumoniae and $C$. pneumoniae are pathogens typically associated with community-acquired pneumonia and are acquired by person-to-person transmission [4]. Standard microbiological work-up of respiratory cultures does not allow for the detection of these pathogens since special media or cell culture are required to grow these fastidious pathogens. Because previous studies investigating the epidemiology of pathogens in HAP and VAP did not account for this, the true incidence of M. pneumoniae and C. pneumoniae may have been underestimated $[5,6]$. So far, data have been primarily limited to case series and studies using serology for the 
detection of these pathogens [7, 8]. Louie et al. performed IgM serology for M. pneumoniae and Chlamydia species in 135 patients with nosocomial pneumonia and identified 1 case each $(0.7 \%)$ [8]. Serology, however, is hampered by several shortcomings, including unclear antibody kinetics and an IgM response to $M$. pneumoniae which may be nonspecific or even absent, particularly in adults. Furthermore, testing antibodies to detect $C$. pneumoniae is not recommended anymore due to insufficient specificity. The use of novel molecular-microbiological diagnostic tools such as quantitative realtime polymerase chain reaction (PCR) technology might overcome these obstacles allowing for a more reliable detection of these pathogens. Aim of the study was to assess the incidence of $M$. pneumoniae and C. pneumoniae in hospital-acquired respiratory tract infections (RTIs) in critically ill patients using PCR technology.

\section{Materials and methods}

This retrospective cohort study was performed in four ICUs (1 medical, 1 neurological, 2 anaesthesiological/surgical) at the Jena University Hospital (JUH). The JUH is the only hospital in the region of Jena, serving approximately 120,000 inhabitants. All ICU-patients $\geq 18$ years with the diagnosis of a RTI who underwent conventional culture techniques and PCR testing (LightMix Modular, TIB MOLBIOL, Berlin, Germany) for both M. pneumoniae and C. pneumoniae from respiratory tract specimens (bronchoalveolar lavage or tracheobronchial aspirates) between January 2013 and May 2017 were included. Testing was ordered at the discretion of the treating physicians. RTI was defined as either CAP (community acquired pneumonia), HAP, VAP or VAT (ventilator associated tracheobronchitis). Diagnosis of CAP, VAP and HAP was established according to accepted criteria [2, 3, 9]. VAT has been defined as fever with no other recognizable cause, purulent tracheal secretions, positive endotracheal aspirate $\left(>10^{5} \mathrm{CFU} / \mathrm{mL}\right)$, and absence of new infiltrates on chest X-ray. The study was approved by the institutional review board.

\section{Results}

Of 552 patients with an established diagnosis of a RTI, 238 (43.1\%) RTIs were community acquired and 314 (56.9\%) hospital-acquired. Only patients with hospital-acquired RTIs were included in further analyses (Table 1). Of these, $210(66.9 \%)$ patients were diagnosed with HAP, 65 (20.7\%) with VAP and 39 (12.4\%) with VAT. Overall, 73 (30.7\%) patients were on mechanical ventilation on the day of microbiological examination. The median day of microbiological examination was day 13 after hospital admission and in patients with VAP on day 6 of mechanical ventilation. Blood cultures were drawn in 307 (97.8\%) patients and were positive in 27 patients (8.8\%). Conventional culture techniques

Table 1 Characteristics and outcomes of patients with hospital-acquired respiratory tract infection

\begin{tabular}{|c|c|c|c|c|}
\hline Characteristics and outcomes & Overall $(n=314)$ & $\operatorname{VAP}(n=65)$ & $\operatorname{HAP}(n=210)$ & VAT $(n=39)$ \\
\hline Male, $n(\%)$ & $231(73.6)$ & $45(69.2)$ & $156(74.3)$ & $30(76.9)$ \\
\hline Age (years), mean \pm SD & $61.7 \pm 14.2$ & $60.0 \pm 14.3$ & $62.0 \pm 13.6$ & $62.6 \pm 16.5$ \\
\hline Immunosuppression, $n(\%)$ & $82(26.1)$ & $7(10.8)$ & $71(33.8)$ & $4(10.3)$ \\
\hline Diabetes mellitus, $n(\%)$ & $102(32.5)$ & $15(23.1)$ & $68(32.4)$ & $19(48.7)$ \\
\hline COPD, $n(\%)$ & $52(16.6)$ & $11(16.9)$ & $34(16.2)$ & 7 (17.9) \\
\hline \multicolumn{5}{|l|}{ ICU type, $n(\%)$} \\
\hline Medical & $137(43.6)$ & $24(36.9)$ & $96(45.7)$ & $17(43.6)$ \\
\hline Anaesthesiological (cardiothoracic) & $74(23.6)$ & $8(12.3)$ & $57(27.1)$ & $9(23.1)$ \\
\hline Anaesthesiological (other surgical) & $95(30.3)$ & $32(49.2)$ & $52(24.8)$ & $11(28.2)$ \\
\hline Neurological & $8(2.5)$ & $1(1.5)$ & $5(2.4)$ & $2(5.1)$ \\
\hline Mechanical ventilation $^{\mathrm{a}}$ (days), median (Q1, Q3) & $11(4.5,21.0)$ & $16(12.0,25.5)$ & $8(3.0,19.0)$ & $14(5.0,28.8)$ \\
\hline Length of hospital stay (days), median (Q1, Q3) & $33(22.0,52.0)$ & $28(21.5,40.0)$ & $35(22.0,52.3)$ & $34(22.0,47.0)$ \\
\hline Length of ICU stay (days), median (Q1, Q3) & $19(10.0,30.3)$ & $25(17.0,33.0)$ & $17(8.8,29.0)$ & $20(14.0,32.0)$ \\
\hline Sepsis, $n(\%)$ & $87(27.7)$ & $21(32.3)$ & $61(29.0)$ & $5(12.8)$ \\
\hline Septic shock, $n(\%)$ & $84(26.8)$ & $13(20.0)$ & $71(33.8)$ & $0(0)$ \\
\hline Hospital mortality, $n(\%)$ & $159(50.6)$ & $24(36.9)$ & $121(57.6)$ & $14(35.9)$ \\
\hline ICU mortality, $n(\%)$ & $139(44.3)$ & $23(35.4)$ & $107(51.0)$ & $9(23.1)$ \\
\hline
\end{tabular}

$C O P D$ chronic obstructive pulmonary disease, HAP hospital-acquired pneumonia, ICU intensive care unit, VAP ventilator-associated pneumonia, VAT ventilator-associated tracheobronchitis, $Q 1$ 1st quartile, $Q 3$ 3rd quartile, $S D$ standard deviation

${ }^{\mathrm{a}}$ Assessed for patients with ventilation only (overall: 302, VAP: 65, HAP: 198, VAT: 39) 
revealed at least one pathogen in $83(26.4 \%)$ patients. The most common pathogens were Enterobacteriaceae $(n=49$, $59.0 \%)$, Staphylococcus aureus $(n=21,25.2 \%)$ and Pseudomonas aeruginosa $(n=12,14.4 \%)$. PCR-testing for $M$. pneumoniae was positive in two patients $(0.6 \%)$ and for $C$. pneumoniae in zero patients. The first patient tested positive for M. pneumoniae was a 56-year-old female patient with acute myeloid leukemia in neutropenia. She was transferred to ICU on day 32 after hospital admission due to HAP with progredient respiratory failure. On day 5 after ICU admission mechanical ventilation was initiated and PCR testing of M. pneumoniae in BAL specimen was performed which was positive. Conventional microbiology in this patient revealed no pathogen. Therapy with clarithromycin was added to the already ongoing therapy with a $\beta$-lactam agent. The patient died on day 21 after ICU admission. The second patient was a 46-year-old female neurosurgical patient without immunosuppression or serious illnesses. She had been admitted to hospital with subarachnoidal bleeding and mechanical ventilation was initiated on the day of admission. On day 4 after admission the diagnosis of VAT was established. M. pneumoniae was detected by PCR in tracheobronchial aspirate. Conventional microbiology revealed no pathogen. Therapy with Clarithromycin was administered for 6 days. The patient was discharged after 4 weeks from hospital. In both patients the positive PCR test result for M. pneumoniae was confirmed by a second PCR testing within 1 week after primary diagnosis.

\section{Discussion}

In this study, in critically ill patients with hospital-acquired RTIs no infection with $C$. pneumoniae was detected and only two patients were tested positive for M. pneumoniae, corresponding to an incidence of $0.6 \%$. Until now, only Apfalter et al. [10] performed a prospective monocentric study using PCR technology for determining the incidence of, amongst other pathogens, M. pneumoniae and C. pneumoniae in patients with hospital-acquired VAP. In this prospective study, 100 patients from medical ICUs with proven VAP hospitalized for $\geq 14$ days were enrolled. $M$. pneumoniae and C. pneumoniae were detected in 3 (3.0\%) and $2(2.0 \%)$ patients, respectively. Hence, an equal low incidence as observed in our study. Interestingly, in our study in patients with CAP, C. pneumoniae and M. pneumoniae was also tested positive in only one patient each, supporting the notion that these pathogens rather cause mild pneumonia not requiring ICU admission [6]. Due to the retrospective nature of the present study, various limitations have to be considered. First of all, as PCR testing was ordered at the discretion of the treating physician cases who did not undergo PCR testing remain unnoticed and incidence might, therefore, be false low. In addition, the generalizability of our findings is unknown because our study was limited to a single healthcare facility. Furthermore, an earlier study showed that persistent carriage of $M$. pneumoniae DNA in the respiratory tract is common following an acute infection. Median time for carriage of $M$. pneumoniae DNA in this study was 7 weeks after disease onset (range 2 days- 7 months) and adequate antibiotic treatment did not shorten the period of persistence [11]. Therefore, it is not for sure that the positive PCR testing for M. pneumoniae in our patients reflects the responsible pathogen for the current episode of RTI.

Nevertheless, considering our results and the results of previous studies the risk of acquiring a hospital-acquired RTI with M. pneumoniae and C. pneumoniae as pathogens seems negligible.

The results of our study support the recommendation of the guidelines not to perform specific microbiological testing and empiric antibiotic therapy covering these pathogens, in particular, taking possible side effects, including cardiotoxicity with macrolide antibiotics, and additional costs for specific microbiological testing into account. Specific microbiological testing should be limited to cases with treatment failure.

\section{Compliance with ethical standards}

Conflict of interest The authors declare that they have no competing interests relevant to the manuscript.

\section{References}

1. Torres A, Niederman MS, Chastre J, et al. International ERS/ ESICM/ESCMID/ALAT guidelines for the management of hospital-acquired pneumonia and ventilator-associated pneumonia: guidelines for the management of hospital-acquired pneumonia (HAP)/ventilator-associated pneumonia (VAP) of the European Respiratory Society (ERS), European Society of Intensive Care Medicine (ESICM), European Society of Clinical Microbiology and Infectious Diseases (ESCMID) and Asociación Latinoamericana del Tórax (ALAT). Eur Respir J. 2017;50:1700582.

2. Kalil AC, Metersky ML, Klompas M, et al. Management of adults with hospital-acquired and ventilator-associated pneumonia: 2016 clinical practice guidelines by the Infectious Diseases Society of America and the American Thoracic Society. Clin Infect Dis. 2016;63:e61-111.

3. Dalhoff K, Abele-Horn M, Andreas S, et al. Epidemiologie, diagnostik und therapie erwachsener patienten mit nosokomialer pneumonie-update 2017. Pneumologie. 2018;72:15-63.

4. Dumke R, Schnee C, Pletz MW, et al. Mycoplasma pneumoniae and Chlamydia spp. infection in community-acquired pneumonia, Germany, 2011-2012. Emerg Infect Dis. 2015;21:426-34.

5. Leroy O, Giradie P, Yazdanpanah Y, et al. Hospital-acquired pneumonia: microbiological data and potential adequacy of antimicrobial regimens. Eur Respir J. 2002;20:432-9.

6. Park DR. The microbiology of ventilator-associated pneumonia. Respir Care. 2005;50:742-63 (discussion 763-5). 
7. Casalta JP, Piquet P, Alazia M, et al. Mycoplasma pneumoniae pneumonia following assisted ventilation. Am J Med. 1996;101:165-9.

8. Louie M, Dyck B, Parker S, et al.: Nosocomial pneumonia in a Canadian tertiary care center: a prospective surveillance study. Infect. Control Hosp. Epidemiol. 1991;12:356-63.

9. Ewig S, Höffken G, Kern WV, et al. Management of adult community-acquired pneumonia and prevention-update 2016. Pneumologie. 2016;70:151-200.
10. Apfalter P, Stoiser B, Barousch W, et al. Community-acquired bacteria frequently detected by means of quantitative polymerase chain reaction in nosocomial early-onset ventilator-associated pneumonia. Crit Care Med. 2005;33:1492-8.

11. Nilsson AC, Björkman P, Persson K. Polymerase chain reaction is superior to serology for the diagnosis of acute Mycoplasma pneumoniae infection and reveals a high rate of persistent infection. BMC Microbiol. 2008;8:93. 